\title{
La ciencia en la Educación Sexual Integral: Aportes desde la epistemología y la pedagogía feminista.
}

The science in the Argentine's sexual education law: Contributions from epistemology and feminist pedagogy

\author{
Catalina González del Cerro*
}

\section{Resumen}

El trabajo desarrolla un análisis teórico sobre el vínculo entre los elementos centrales de la epistemología feminista y su despliegue en el ámbito educativo argentino. Se analizarán los tres proyectos feministas elaborados por Sandra Harding -empirista, punto de vista y posmoderno- a la luz de algunas preguntas que la pedagogía feminista viene desarrollando. Asimismo se analizarán algunos documentos que se desprenden de la Ley Nacional 26150 de Educación Sexual integral (2006) y en particular sus sentidos en torno a la objetividad científica. Se busca aportar al campo de la educación y el género a partir de describir las tensiones que aparecen entre la conceptualización escolar de la naturaleza y los cuerpos, y la problematización del determinismo biológico que introduce la teoría feminista en el ámbito escolar.

Palabras claves: Educación sexual - Epistemología feminista - Género - Pedagogía Androcentrismo.

\begin{abstract}
The paper develops a theoretical analysis on the link between the central elements of feminist epistemology and its deployment in the Argentine educational field. The three feminine projects elaborated by Sandra Harding - empiricist, point of view and postmodern - will be analyzed in the light of some questions that the feminist pedagogy has been developing. It will also analyze some documents that come out of National Law 26150 of "Educación Sexual Integral" (2006) and in particular their senses around scientific objectivity. In this way, we seek to contribute to the field of education and gender by describing the tensions that appear between the school conceptualization of nature and bodies, and the problematization of biological determinism introduced by feminist theory in school.
\end{abstract}

\footnotetext{
* Es Doctoranda por la Facultad de Filosofía y Letras con orientación en Educación por la Universidad de Buenos Aires. Es Becaria doctoral del Consejo Nacional de Investigaciones Científicas y Técnicas (CONICET) y Profesora de nivel superior y medio en Ciencias Antropológicas. Actualmente es investigadora del proyecto Ubacyt "Transversal y con contenidos propios" Educación sexual con enfoque de género en la formación docente inicial" dirigido por la Dra Graciela Morgade. Argentina. E-mail: catalinagc@gmail.com
} 
Keywords: Sexual education - feminist epistemology - gender - pedagogy androcentrism.

Fecha de recepción: 21 de marzo 2017

Fecha de aprobación: 20 de enero 2018

\section{Introducción}

La Ley de Educación Sexual Integral (en adelante ESI), en los más de 11 años que tiene de vigencia en la Argentina, ha dejado planteado un punto de partida específico, un "desde dónde" comenzar a enseñar sobre sexualidad y género. La ley (26150/2006) surge, en gran medida, en respuesta a la escasa y fragmentada normativa preexistente en el país y a una tradición que silenciaba los temas o enfoques referidos a los cuerpos sexuados $\mathrm{o}$, si los explicitaba, lo hacía desde sentidos moralizantes, preventivos y amenazantes de la sexualidad, principalmente la juvenil. Frente a estos antecedentes, los documentos de la política ESI proponen, entre otros elementos, un abordaje de la educación sexual con perspectiva de género, y también, la necesidad de un "perspectiva científica" de la educación sexual ${ }^{1}$, de modo de hacerse cargo de expresiones menos rigurosas sobre los cuidados del cuerpo, muchas veces sostenidas por discursos sexistas o "mitos" que provienen de sentidos comunes sedimentados.

En cuanto al primero punto, la inclusión de una mirada crítica sobre las desigualdades de género se nutrió en gran parte, y sobre todo en los últimos años, de los aportes teóricos de las pedagogas que vienen investigando desde la óptica feminista y de un intercambio más fluido con los activismos de género y con experiencias pedagógicas previas. Sin embargo, en relación a lo segundo, la adscripción sin reparos a un abordaje científico de la sexualidad en los textos parece asumir una idealización de la ciencia como ámbito homogéneo y neutral. Estas citas de autoridad científica desconocen los numerosos debates al interior de los feminismos, y de la epistemología feminista en particular, en torno a las formas en que se expresa el sesgo androcéntrico en la conformación de las comunidades científicas, en los problemas que se deciden investigar y financiar, y en los resultados de estas investigaciones, que luego se sintetizan en el currículum escolar.

A partir de identificar esta tensión presente en la normativa, en este artículo nos proponemos reconstruir, en un primer momento, los principales debates que contemplan

\footnotetext{
${ }^{1}$ Nos referimos principalmente a los "Lineamientos curriculares ESI" de alcance nacional que explicita como derecho de Ixs estudiantes a aprender sobre "la sexualidad humana desde la perspectiva científica" (LNC 2008: 36). En el texto de la ley de nación no aparece mención ni a una perspectiva de género ni a una perspectiva científica, pero si se mencionan ambas en la Ley ESI (22100/2006) de la Ciudad Autónoma de Buenos Aires, que plantea entre sus objetivos del artículo 5 "Brindar información científica, precisa, actualizada y adecuada a cada etapa de desarrollo de los alumnos/as, acerca de los distintos aspectos involucrados en la Educación Sexual Integral." y en su artículo 4to " El reconocimiento de la perspectiva de género en los términos del art. 38 de la Constitución de la Ciudad Autónoma de Buenos Aires".
} 
tanto el campo de la epistemología como de la pedagogía feminista con sus especificidades disciplinares, para luego ponerlos a dialogar y poder identificar puntos de encuentro que resulten útiles a futuras líneas de investigaciones empíricas en torno a los modos de traducción o apropiación de la perspectiva de género en las instituciones. Para ello, retomo la categorización que elabora una teórica de la ciencia que, según considero, permite iluminar y complejizar algunos interrogantes propios del ámbito educativo.

En un segundo momento, busco incluir la pregunta por los posibles proyectos feministas en la pedagogía, una reflexión que incorpore la dimensión de las políticas educativas, en concreto en relación a la ESI. Así busco complejizar estos debates al circunscribirlos en contextos legales particulares en los que son necesarios consensos pedagógicos y sociales que puedan ir más allá de los esfuerzos individuales que algunas educadoras realizan para llevar el feminismo a sus aulas. Por eso me detengo, a partir de la técnica de relevamiento de documentos, en los sentidos que la normativa ESI despliega en algunos de sus textos en torno la ciencia, la naturaleza y los cuerpos, categorías que toman protagonismo en los debates teóricos planteados en el primer momento. Para ello opto por limitar mi indagación a los fragmentos de los documentos referidos a la asignatura Ciencias Naturales.

Esta artículo se enmarca asimismo en un proyecto de investigación doctoral más amplio que se pregunta por las modalidades en que se transversaliza la perspectiva de género en las escuelas secundarias de la Ciudad Autónoma de Buenos Aires. De allí que recurro de forma más escueta a algunas hipótesis nutridas de mis observaciones del trabajo de campo etnográfico desarrollado en los últimos 5 años $^{2}$.

\section{La epistemología feminista}

La objetividad y neutralidad de la ciencia positivista han sido largamente cuestionadas por la epistemología crítica, que elaboró una rigurosa lectura política de las características atribuidas al sujeto epistémico de la ciencia. La sociología de la ciencia, que se desplegó con mayor profundidad a partir de la década del 60, focaliza en la pertenencia de Ixs científicxs $^{3}$ a una clase social y a un grupo étnico dominante, así como también en su actitud conservadora en defensa de los privilegios de la élite científica y social. A su vez, el campo de la historia de la ciencia ilumina el carácter procesual y relacional de la teorías científicas, y pone en duda sus pretensiones de universalidad.

Acompañando estos desarrollos de la teoría crítica, la epistemología de inspiración feminista (Fox Keller, 1991; Haraway, 1995; Harding, 1996; Schiebinger, 2004) denunció tempranamente el carácter sesgado de la ciencia positivista y reforzó el cuestionamiento

\footnotetext{
${ }^{2}$ Este consistió en observaciones participantes y entrevistas en profundidad a docentes, directivxs y estudiantes de dos escuelas secundarias de la Ciudad Autónoma de Buenos Aires, así como también a funcionarias y capacitadoras de ESI en la ciudad.

${ }^{3}$ En este trabajo optamos por usar la flexión de género en " $x$ ", que apunta a contrastar críticamente el protocolo hegemónico de la construcción masculina del sujeto universal.
} 
al ideario moderno que deposita la esperanza del bienestar en el control de la naturaleza y en un progreso lineal e ilimitado. Estos cuestionamientos comenzaron a emerger junto con la cada vez mayor presencia de mujeres en los estudios superiores, y una progresiva inserción laboral de éstas en las comunidades científicas (Bonder, 1994). De la mano de estas primeras académicas se desarrollaron los estudios de la mujer - que luego pasaron a denominarse "estudios de género"-, y se apuntó al campo científico como uno de los ámbitos sociales en los que se reproducen las normas, valores y expectativas diferenciales propios del orden patriarcal (González García y Pérez Sedeño, 2002).

Tal como lo sistematizan Marta González García y Eulalia Pérez Sedeño, (2002) en los años que se corresponden con la segunda ola del feminismo se elaboraron numerosos textos biográficos dedicados a reescribir la historia oficial y "rescatar" a aquellas mujeres que hicieron contribuciones relevantes a la corriente principal de la ciencia. Mujeres que han estado invisibilizadas en el relato oficial y en un gran porcentaje de los casos no tuvieron los mismos laureles simbólicos y materiales que sus colegas o sus maridos. Sin duda estos trabajos fueron muy importantes para mostrar que no sólo no hay una limitación cognitiva para el ejercicio de las ciencias, sino también para dar cuenta de los múltiples e injustos esfuerzos que estas científicas tuvieron que realizar para poder desarrollar sus trabajos y obtener reconocimiento. Asimismo, otros estudios enfocados en la historia de la tecnología (Pérez Sedeño, 1998) han denunciado que aquellos inventos realizados por mujeres y relacionados con la esfera de lo doméstico y la crianza, no han sido debidamente reconocidos como desarrollos "tecnológicos".

En sintonía con las advertencias del feminismo de la diferencia (Irigaray y Alonso, 1978; Braidotti, 2004), la búsqueda de reconocimiento y jerarquización en el ámbito científico por parte de mujeres posee como contracara el riesgo latente de retener las normas masculinas como medida de excelencia. Es decir, se advierte la tendencia a reemplazar a los "héroes" por "heroínas", como casos excepcionales e individuales de aquellas "que supieron" sortear los obstáculos y alcanzar los privilegios de los que ya gozaban los varones. El ejercicio reflexivo sobre qué sujetos conformaron tradicionalmente la comunidad científica permite poner en evidencia que el producto de esta comunidad -el conocimiento científico- está sesgado por las experiencias subjetivas de aquellos sujetos. Que las mujeres hayamos sido históricamente "objeto" de la ciencia, pero no así sujetas protagonistas de ella, permite comprender el androcentrismo en el campo y en sus prácticas.

Con pensamiento androcéntrico nos referimos a la propensión a considerar tácitamente a los varones como sujetos de referencia (Silvia Elizalde, 2006), y que según Diana Maffía (2007) se enraíza en la tradición aristotélica. Aristóteles, en sintonía con la organización social de la antigua Grecia, establece que no todos los sujetos están en condiciones de elaborar conocimiento válido. No están en condiciones las mujeres, Ixs niñxs y lxs esclavxs, ya que a ellxs se les atribuye capacidades de menor valor epistémico: subjetividad, sensibilidad, singularidad y narratividad. Estos se oponen (siguiendo el mismo orden) a la objetividad, la racionalidad, la universalidad, y la abstracción que, se asumía, sí poseía el 
varón adulto y amo -el andros-, y que derivaron en elementos centrales del pensamiento científico dominante posterior. El esquema, lejos de responder a principios lógicos, se sustenta en características atribuidas a sujetos sociales en relaciones asimétricas de poder. Esta separación se basa en un esquema dicotómico basado en el principio de exhaustividad y exclusividad: se asume que no existen otras opciones por fuera de ese par dicotómico, ni es posible encontrar instancias intermedias.

Uno de los mayores desafíos epistemológicos estaría asociado a una superación del propio esquema dicotómico y jerarquizado que sostiene el pensamiento científico moderno y se corresponde con la construcción binaria de los géneros: cultura/naturaleza, objetividad/subjetividad, raciona/emocional, mente/cuerpo, fuerte/frágil, público/privado. Visto desde el pensamiento androcéntrico, si la Cultura con mayúsculas (entendida como civilización que requiere de la ciencia positiva) representa dimensiones predominantemente masculinas, su contraparte, la naturaleza, sigue estando vinculada en última instancia a la mujer, y a su fertilidad irracional alojado en su útero.

Diana Maffía (2007) describe tres modos existentes de abordar la aún persistente marginalización femenina en el ámbito científico. El primero es el punto de vista conservador, el cual afirma que las mujeres simplemente no pueden hacer ciencia tan bien como los hombres, ya que hay "algo" en su naturaleza física, psicológica e intelectual que las incapacita para producir ciencia. El segundo modo de ver el problema, que suele llamarse liberal, ve la ausencia de mujeres como una cuestión de acceso a la educación y el empleo, y propugna la integración a través de medidas de acción afirmativas y asume una ineludible y eventual igualdad en el acceso. El tercero, un punto de vista radical y que consideramos el más útil a los fines de este análisis, sostiene que no es suficiente para las mujeres ser científicas si la ciencia va a continuar como hasta el presente. Esta es la posición de Sandra Harding (1996) cuando se pregunta ¿deben las mujeres moldear sus valores y métodos para acomodarse a la ciencia, o la ciencia moldeará sus métodos y prácticas para acomodarse a las mujeres?

\section{La pedagogía feminista}

Este breve recorrido por los aportes de científicas feministas brinda herramientas para reflexionar sobre los modos en que se construye y enseña conocimiento sobre los cuerpos al interior de las instituciones escolares y académicas. Así como las feministas tempranamente apuntaron a la ciencia como anclaje de discursos patriarcales, también identificaron que las instituciones educativas modernas, lejos de contrarrestar la desigualdad entre géneros, colabora en su reproducción.

Estas ideas emergieron a la par de nuevas corrientes dentro de la sociología de la educación norteamericana y europea en los años 60 y 70, que se dedicaron a develar la politicidad de las prácticas educativas y a denunciar que la masificación de la escolaridad no basta para lograr mayor igualdad en el orden capitalista. Dentro de estas encontramos, por un lado las llamadas Teorías críticas de la educación que según Luis Rigal (2011) se han 
centrado en la escuela y el currículum, pivoteando sobre las nociones de hegemonía y resistencia (Willis 1983; Apple 1985; Giroux 1992), con fuerte influencia del pensamiento gramsciano, y de violencia simbólica (Bourdieu y Passeron, 1979; Bernstein, 1990). Por otro lado, emergen en el contexto latinoamericano las Pedagogías críticas (Freire 1970; Shor, 1980) enfocadas en las relaciones de poder propias del vínculo pedagógico, desarrollando líneas orientadoras para una práctica docente que enfatice en las categorías de autonomía, criticidad, diálogo y liberación.

Si bien la perspectiva de género no ganó legitimidad en ese campo hasta entrados los años 80, estos trabajos aportaron herramientas para analizar las contribuciones del sistema educativo a la reproducción y legitimación de las múltiples desigualdades. Por ejemplo, estos trabajos aportaron a la definición de conocimiento escolar como una construcción formalizada, diferente de la que los campos mismos de las ciencias académicas han separado, lejos de ser neutrales, los campos disciplinares son escenarios de lucha, con reglas de inclusión y exclusión orientadas por fuentes de prestigio y de financiamiento mucho menos maleables que el currículum explícito escolar.

Tanto para la sociología de la ciencia y de la educación, el "qué" y el "cómo" se investiga es una decisión adoptada en ámbitos académicos: aquello que se investiga en los institutos de las facultades, que se difunde en revistas nacionales e internacionales, que se ramifica en especialidades y que es permanentemente debatido por personas que constituyen la "comunidad científica". Qué se enseña de lo que se investiga es una decisión que toman los equipos profesionales técnicos de las áreas de política curricular central y distingue en secuencia y gradualidad según niveles. Por último, qué se enseña en cada escuela y/o aula es otro nivel de decisión, en el cual los miedos, las expectativas y los saberes docentes tienen una incidencia mayor.

Inspiradas en esta mirada crítica, distintxs investigadorxs feministas (en Inglaterra, Epstein y Johnson, 1998; en Canada, Britzman, 1999; en Brasil, Lopes Louro, 1999) han dado cuenta de que lo comúnmente enseñado como contenido en las asignaturas, expresa una naturalización de las normas de género. De la mano de nuevos interrogantes propios de los Estudios de género, el campo de la pedagogía feminista fue mutando y combinando sus objetos de estudio: ya no se trata de constatar únicamente desigualdades en términos del diferencial acceso a la educación, sino también de entender cuáles son los mecanismos que constituyen a la escuela como espacio sexista y heteronormativo.

En la Argentina y de forma más reciente, diversas investigaciones del campo de educación, género y sexualidades (Morgade1995, 2006, 2011; Scharagrodsky, 2008; Tomasini 2011; Pechin 2012;Baez, 2013;Alonso y Zurbriggen, 2014; Molina 2014) se han dedicado a describir los múltiples dispositivos de disciplinamiento que la escolarización realiza sobre los cuerpos de estudiantes y docentes en relación a las distinciones y atribuciones deseables de masculinidad y feminidad. Las expectativas diferenciales, los discursos y las prácticas dentro y fuera del aula dejan, según estos estudios, marcas profundas, 
cotidianas y sutiles en niñxs y jóvenes, y son las que logran hacer mella de manera más duradera en la experiencia escolar, por no estar ni explicitadas ni reconocidas.

A diferencia de los escritos del equipo de Claudia Korol (2007; 2016) y Luz Maceira Ochoa (2008) que posicionan a "pedagogía feminista" como vinculada a las propuestas desplegadas en espacios educativos barriales de los movimientos sociales, la definición tomada aquí se posiciona en y desde la escuela pública. Incorpora elementos propios de los estudios que identifican los sesgos androcéntricos de las fuentes disciplinares del currículum (Pacheco Ladrón de Guevara, 2010; Morgade, 2013; Da Cunha, 2015), como también incorpora los saberes producidos en la práctica política de los movimientos feministas y de la disidencia sexual. Algunos características que habilitan posibles abordajes desde la práctica escolar son, tal como expresa Graciela Morgade (2011, 2013), la aproximación a la experiencia como fuente válida, el ejercicio de la propia voz, la discusión de la autoridad y las fuentes de autorización, la centralidad de la posición antes que la esencialización de las relaciones de poder.

\section{Tres miradas sobre las mujeres en la educación}

Vimos entonces que la pedagogía feminista genera un aporte sustancial en la consolidación de una metodología propiciadora de relaciones más igualitarias en términos de género, al tiempo que produce condiciones más justas de producción de saberes. Esta metodología dialoga con los planteos que hacen las epistemólogas: es necesario poner en valor social la experiencia subjetiva de las mujeres y la posibilidad de enunciar desde el lugar de subordinación, como modo de combatir o contrarrestar a una autoridad epistémica basada en una supuesta objetividad y neutralidad.

En este trabajo diremos que la paradoja que identifica y desarrolla la epistemóloga Sandra Harding (1996) en relación a los límites de la ciencia es susceptible de ser analizada desde la pedagogía para analizar las instituciones escolares: ¿Es posible utilizar con fines emancipadores una educación tan íntimamente inmersa en los proyectos androcéntricos? Para profundizar en el dialogo entre estos dos campos recién abordados, intentaremos responder esta pregunta utilizando el esquema que realiza Harding sobre tres proyectos feministas en torno al conocimiento - el empirismo, el del punto de vista y el posmodernoa la luz de algunas lecturas que son propias de la pedagogía. A continuación, repasaremos la caracterización que realiza la autora de estos tres proyectos que, con sus respectivas debilidades, aportan a nuestro análisis y amplían la posibilidad de complejizar las respuestas desarrolladas por las pedagogas en torno al androcentrismo escolar.

\subsection{Empirismo}

El primer proyecto sostiene que el sexismo y el androcentrismo constituyen sesgos sociales corregibles mediante la estricta adhesión a las normas metodológicas vigentes de la investigación científica. Más cercano al planteo del feminismo de la igualdad, este proyecto expresaría que en la medida en que haya más mujeres científicas es más 
probable que la centralidad masculina disminuya, y se perfeccionen los métodos para alejarse cada vez más de la "ciencia mala", es decir sesgada. Este error estaría siendo paulatinamente corregible, a partir de acciones que tiendan a promover la igualdad de oportunidades de acceso o de políticas de cupo femenino.

Es indiscutible que en el ámbito científico el porcentaje de mujeres fue creciendo en las últimas décadas. También es cierto que en la actualidad las mujeres siguen siendo minoría y permanece un imaginario basado en representaciones estereotipadas de las capacidades intelectuales femeninas. De acuerdo a las cifras relevadas en 2012 por el Instituto de Estadísticas de la UNESCO ${ }^{4}$, el 30\% de la comunidad científica a nivel mundial son mujeres. Además, en el ámbito científico la participación de las mujeres en funciones de dirección sigue siendo muy limitada y también se mantiene la segregación "horizontal", restringiendo su participación en ciertas actividades predominantemente masculinas. La desigualdad se ve aún más en relación a las condiciones de trabajo, así como también en las oportunidades para mantener una formación continua y en la creación de una red de contactos.

Sandra Harding señala un aspecto negativo de este proyecto empirista, que sólo destina esfuerzos a la dimensión metodológica, se aplica al contexto de justificación y no al contexto de descubrimiento. Esto es un problema, ya que un origen clave del sesgo androcéntrico se sitúa en la selección de los problemas de investigación y en quiénes y cómo definen un fenómeno dado como problema que amerita ser investigado.

Si hacemos un paralelo para el ámbito educativo, aparece la siguiente pregunta ¿si la docencia, a diferencia de la actividad científica, es un ámbito primordialmente femenino desde los inicios de la escuela argentina, no debería ello garantizar una mayor reflexividad sobre el androcentrismo escolar, o sobre las injusticias del patriarcado? El trabajo docente representa un claro contraejemplo de esta hipótesis empirista: si bien en términos porcentuales el trabajo docente es ampliamente femenino desde hace más de un siglo, fue también un ámbito particularmente resistente a las prácticas y discursos de los feminismos.

Durante décadas la feminización del trabajo docente estuvo tan íntimamente atravesada por una matriz patriarcal que -hasta hace pocos años- mantuvo vedada la problematización de los contenidos sexistas enseñados por estas mismas docentes. Distintxs autorxs vinculan el imaginario social de la maestra como segunda mamá, abnegada y asexuada y su rol como "agente civilizatorio" que impregnó el normalismo de principio de siglo (Finocchio, 2009). Limitándose a trasmitir una cultura heredada de

\footnotetext{
4 "Women in sciencie", UNESCO Institute for Statistics. Noviembre, 2015. №34. Consultado: 1/10/2017 en http://uis.unesco.org/sites/default/files/documents/fs34-women-in-science-2015en.pdf. Se destaca en este informe que Argentina es uno de los países con mayor porcentaje de mujeres trabajando activamente en ciencia del mundo, siendo un porcentaje de $52 \%$
} 
forma amorosa, las docentes siguen encontrando muchas dificultades para encarnar como sujeto oprimido y enseñar desde ese punto de vista politizado.

El problema no se limita a una cuestión de acceso, sino resulta necesario ahondar en la persistencia de modelos pedagógicos que asumen que la posición social, el género o a la cantidad de personas que transitan las aulas no debería afectar la tarea docente o aquello que se enseña. Esto se basa en falsos supuestos, como expone Flavia Terigi (2000), considerando posible una fidelidad del currículum al saber, y una fidelidad de la escuela al currículum. Esta lectura aséptica del contenido escolar, fuertemente criticado por la sociología de la educación, aparece directamente vinculada a la supresión de la subjetividad de docentes y estudiantes, de sus cuerpos, sus conflictos, sus deseos.

Al igual que con el imaginario de la maternidad, la "señorita maestra" encuentra los fundamentos de su rol cuidador en la esfera de lo instintivo y/o vocacional. Graciela Morgade (2010) analiza a su vez el sentido paradojal de la tarea de las mujeres directoras y supervisoras escolares ya que, si bien el trabajo docente en el aula ha sido signado como "femenino" desde fines del siglo XIX, la asunción de cargos de poder por parte de las mujeres solo recientemente devino un derecho legítimo y ejercido. Una empirista dirá que afirmaciones tales como "las mujeres son mejores cuidadoras por su capacidad gestante" son verificablemente erróneas por su tinte esencialista y apuntará al sesgo presente en el método y en el contexto de justificación. Pero, ¿no será, como dice Harding, que el problema radica en que justamente no se identifica en estas frases algo problemático a desentrañar, a desaprender? Como nos indican las reflexiones propias de la epistemología crítica, la selección y definición de los problemas "socialmente relevantes", los que luego se traducen en resultados científicamente comprobados y finalmente en conocimientos escolarizables, llevan consigo las huellas de los grupos dominantes de una cultura.

\subsection{Punto de vista feminista}

Esto nos lleva al segundo proyecto que plantea: Es científica y moralmente preferible que las investigadoras sean feministas. La posición dominante de los hombres en la vida social se traduce en un conocimiento parcial y sesgado, mientras que la posición subordinada de las mujeres habilita la posibilidad de un conocimiento más completo y menos perverso. A diferencia de la aspiración del empirismo de que la identidad social del investigadorx no influya en la objetividad deseable, para el punto de vista feminista, esta identidad resulta central, ya que descree de toda neutralidad. Si esquematizamos este proyecto con las corrientes de los feminismos, podemos asociar a esta mirada con los planteos antiasimilacionistas característicos del Feminismo de la diferencia, que buscó valorizar e incluso jerarquizar el lugar de enunciación de las mujeres.

Sin embargo, hay dos fuertes cuestionamientos a esta idea. Por un lado, la imposibilidad de hablar de un mismo punto de vista feminista, ya que la experiencia social de las mujeres (y también de las feministas) está atravesada por otras variables de clase, raza, edad, etc. Por otro lado se corre el riesgo de caer en un esencialismo identitario. Un punto 
importante que la autora defiende es que el compromiso con los valores antiautoritarios fomenta una mayor rigurosidad en las producciones científicas. Despegándose de posiciones relativistas, Harding aclara que "son los movimientos de liberación social los que más han aumentado la objetividad de la ciencia y no las normas de la ciencia misma cuando se han puesto en práctica" (1996: 24).

Si trasladamos este debate a la educación ¿la pedagogía feminista requiere necesariamente de una identidad política específica y explicitada de Ixs docentes? Lejos de resolverse, esta pregunta sigue abriendo polémica en los ámbitos escolares. Las pedagogías críticas, al afirmar que toda educación es política, también desplegaron distintos niveles de responsabilidad a individuos, instituciones y al Estado. Estos debates aparecen con mayor fuerza en relación a asignaturas comúnmente asociadas con contenidos leídos como éticos, ideológicos o políticos, como es el caso de Historia o Formación ciudadana, y mucho menos cuestionados en las otras de mayor jerarquía en la escala de legitimidad (las ciencias -no casualmente llamadas "exactas").

Más allá de la normativa ESI, existen y han existido docentes comprometidxs con promover la reflexión sobre injusticias sociales, y más recientemente, sobre las desigualdades de género ¿Qué sucede cuando unx profesorx o maestrx se dice feminista? ¿Eso requiere que historice y contextualice la teoría y la práctica feminista? ¿Es necesario, viable y/o deseable que se inscriban en una trayectoria militante? Si bien han habido numerosas investigaciones en las últimas dos décadas centradas en las representaciones de la población docente en torno a la educación sexual (Kornblit, Sustas, y Adasko, 2013; Faur et al, 2015), son aún escasos los trabajos que se enfocan en experiencias escolares en las que se incorpora de modo explícito la perspectiva feminista en la enseñanza y en el aprendizaje.

Por otra parte ¿cómo se "transversaliza" el enfoque de género o feminista en las distintas asignaturas? ¿Qué significa enseñar desde un punto de vista feminista? Los trabajos de la norteamericana Peggy Mcintosh (1990) representan una referencia ineludible frente a esta pregunta. Describe una serie de "fases interactivas del desarrollo curricular", advirtiendo que éstas no deben entenderse con un linealidad cronológica sino que usualmente se superponen. Sintéticamente diremos que una primer "fase" corresponde a la invisibilización de las mujeres (y agregamos, las disidencias sexuales) en tanto sujetas de la ciencia, de la historia o de cualquiera de las asignaturas a la que es aplicable este modelo. Una segunda y tercera fase refieren al esfuerzo por "sumar" o añadir a aquellas personas o aquellos problemas que despiertan su ocultamiento. Es decir, elaborar una historia compensatoria, un rescate de las "figuras extraordinarias" de los grupos excluidos. Podríamos identificar en esta fase el optimismo que caracteriza también al empirismo feminista. La tercera y cuarta fase propuesta, que vínculo con el "punto de vista feminista", traslada el foco desde "el caso" hacia el problema social, cuestionando fuertemente las desigualdades de género a nivel estructural. Aparece aquí una verdadera revisión epistemológica y política del currículum y una denuncia a las exclusiones, así como también una visibilización de las luchas de los diferentes grupos subordinados. 


\subsection{Posmodernismo feminista}

El tercer proyecto lo denomina "Posmodernismo feminista". Además del escepticismo frente a cualquier enunciado universal (y masculino) que caracteriza al posmodernismo, esta corriente plantea que "las reivindicaciones feministas solo son aceptables y menos deformantes si se basan en la solidaridad entre estas identidades fragmentadas modernas y entre las políticas que crean" (Harding1996: 27). Una primera tensión es que la insistencia por las pluralidades puede devenir en signo de debilidad frente a un contexto en el cual las relaciones de fuerza sexistas, racistas, clasista no son favorables y se muestran unificadas.

La tensión principal que introduce la categoría de diversidad o la multiculturalidad en el campo pedagógico radica en la supuesta incompatibilidad con el objetivo homogeneizador y nacionalista de la escuela tradicional, la escuela normalista. También se complejiza a partir de las transformaciones de las últimas décadas que consolidaron tipos de subjetividades urbanas, modeladas por un modo de producción que requiere altos niveles de incertidumbre y plasticidad (Puigross, 2014). La solidaridad (y la sororidad) parecería ser el reducto último del lazo social, en una época en que las instituciones parecen haber abandonado ese lugar.

La perspectiva feminista requiere necesariamente de una reconfiguración del vínculo pedagógico, basado en la confianza y en la experiencia, sin por ello dejar de fortalecer la responsabilidad de Ixs docentes y de la institución de habilitar espacios protegidos y significativos para un aprendizaje emancipador. En esta línea posmoderna, Deborah Britzman (1999) pregona por un "pedagogía queer" sostenida en la posibilidad de la incertidumbre. Plantea que, una vez cuestionadas las relaciones de poder, esta versión "aun no tolerada" de la pedagogía debe apuntar al cuidado de sí como práctica de la libertad. Tal como observan Maher y Thompson (1994) Ixs profesorxs feministas buscan suelos alternativos donde construir su autoridad como maestrxs" de igual a igual", a partir de una intersubjetividad consensuada y resistiendo los estándares androcéntricos de su socialización profesional. Ven como socialmente relevante su experiencia en la disciplina y en su propio trabajo escolar, no porque produzcan "verdades" objetivas, sino porque este conocimiento modela su desarrollo personal aun en curso.

Los debates académicos sobre el androcentrismo de la ciencia hicieron eco en los esfuerzos de las educadoras feministas, quienes vieron en la escuela un territorio de reproducción y también de resistencia sobre los sentidos hegemónicos de cuerpo, género y deseo. Este cruce, que rescata el esquema de Harding, nos habilita a imaginar tres ejes o categorías posibles para analizar los modos en que se incluye o se han incluido la teoría y las prácticas feministas a las escuelas: i) a partir de revisar el carácter feminizado de la práctica docente y su sistemática exclusión en los espacios de producción ( y no sólo de transmisión) de los saberes considerados como socialmente valiosos, ii) a partir de explicitar frente a la comunidad escolar un posicionamiento o identidad política propia 
como docente, afirmar que todo educador/a/x enseña desde puntos de vistas sesgados, y referenciarse en una genealogía de estudios y prácticas propias de los feminismos, iii) a partir de describir al conocimiento escolar como una producción situada en un contexto temporal y espacial específico y como resultado de una correlación de fuerzas plasmada en desigualdades de género, siempre en intersección con otras opresiones.

A partir de algunas investigaciones referenciadas en etnografías escolares en la Argentina antes citadas, arriesgamos la hipótesis de que, tal como sucede con las fases interactivas de Mcintosh, estos proyectos tienden a superponerse y a convivir en las estrategias que ensayan tanto docentes como instituciones para traducir un enfoque de género y en relación a los condiciones dadas de cada escuela. Ahora bien, ¿qué sucede cuando los distintos esfuerzos de educadorxs y de feministas se plasman en una política pública, como la ESI, que plantea como obligatoria una educación con perspectiva de género?

En el próximo apartado intentaremos delinear algunas respuestas a esta pregunta situándolas en el contexto argentino de principios de siglo XXI y en el marco de la plena vigencia de una política educativa, la Educación Sexual Integral (Ley 26150/2006). Esta, si bien no explicita un punto de vista feminista, tiene entre sus propósitos develar las desigualdades entre varones y mujeres en modo transversal a las asignaturas y a los niveles educativos, y a la vez defiende una "enseñanza científica" de la sexualidad, como resultado de las disputas con las miradas moralistas o religiosas que marcaron la enseñanza tradicional sobre los cuerpos. En esta encrucijada ¿cómo se entiende y cómo se despliega en los textos y documentos de la ley la "perspectiva de género" en relación a la matriz positivista y androcéntrica de los saberes escolares?

En otros trabajos de investigación en escuelas secundarias de la Ciudad de Buenos Aires (González del Cerro y Busca, 2017) encontramos que es en las definiciones de la naturaleza donde radica uno de los "núcleos duros" del androcentrismo escolar y del determinismo biológico que lo sigue. Así, no sólo "lo personal es político", también lo es lo natural. A partir de este axioma a continuación ampliaremos específicamente en el cruce entre la asignatura Ciencias Naturales y los debates de la pedagogía/epistemología feminista recién vista. Esta selección no desconoce, sin embargo, que la construcción social y escolar de la naturaleza no sólo es susceptible de ser examinada en las asignaturas "más científicas" como física, química y biología (y en las disciplinas universitarias que las alimentan), sino que se constituye como un contenido transversal, permeando las distintas instancias y espacios de la vida cotidiana de las instituciones.

\section{La ciencia según la Educación Sexual Integral}

En la Argentina en las últimas décadas se sancionaron diversas políticas que, con distintos grados de conflictividad y participación de organizaciones civiles, apuntaron a la responsabilidad del Estado en la promoción de la igualdad de derechos para mujeres y para el colectivo de gays, lesbianas y personas trans. Respecto al ámbito educativo, en el 2006 se sancionó la Ley Nacional 26.150 de Educación Sexual Integral (ESI), como 
producto de un largo proceso de discusión democrático y en sintonía con una tendencia latinoamericana (Baez, 2016). Esta estuvo dirigida a orientar la elaboración curricular para todos los niveles educativos, incluyendo a la formación docente, y la producción de materiales de apoyo y proyectos de capacitación e intervención pedagógica. El trabajo de Morgade y Alonso (2008) identifica distintas tradiciones o modelos "ideales" sobre el abordaje la educación sexual que convivieron en nuestro país en las últimas décadas: el biomédico, el moralizante, el de la sexología, el judicializante, el de género. Cada modelo da cuenta de distintos modos de caracterizar la sexualidad y los cuerpos, pero fundamentalmente construyen cánones de legitimidades e ilegitimidades sobre los conocimientos acerca de los cuerpos y las sexualidades, así como también prescriben determinados papeles para las instituciones educativas en cuanto a la transmisión de dicho saber. Mientras el biomédico basa su legitimidad en la neutralidad y objetividad del método científico, el moralizante se sustenta bajo la idea arraigada de que los valores más beneficiosos para la sociedad sólo son garantizables bajo el dogma religioso. Ambos enfoques que supieron convivir durante décadas en las escuelas laicas, comparten el refuerzo de un "pensamiento esencialista" de la sexualidad, como lo denominó Jeffrey Weeks hace varias décadas, que además de reducirla a una dimensión ahistórica, la somete al ámbito de lo privado.

Podemos pensar estos enfoques como tradiciones epistemológicas, que permiten comprender el contexto político y académico sobre el que se desplegaron las disputas de sentido en la constitución de la normativa ESI y los acuerdos finales. La puja estuvo protagonizada en gran parte por los sectores vinculados a la iglesia católica, cuyas posiciones en torno a la sexualidad y al rol de las instituciones educativas se veían contrastadas por otros actores de la sociedad civil. Un tema de debate álgido fue, justamente, si debía o no mencionarse el término género, que finalmente no quedó explicitado en la ley de alcance nacional ${ }^{5}$. Sin embargo, sí se la menciona como tal en las leyes educativas provinciales que surgieron luego para reforzar su aplicación y particularmente en las líneas de acción, curso y materiales elaborados durante los siguientes años por parte del Programa Nacional ESI (PNESI).

\subsection{Las ciencias naturales según la ESI}

Los Lineamientos Curriculares Nacionales para la ESI abarcan todas las áreas curriculares y todos los niveles educativos. En el secundario, divide el nivel en Ciclo Básico y Ciclo Orientado, y presenta en cada uno una lista específica de contenidos. Ambos ciclos están

\footnotetext{
${ }^{5}$ En el texto de la ley de alcance nacional podemos encontrar una mención en la formulación del objetivo "Procurar igualdad de trato y oportunidades para varones y mujeres". En la Ley ESI 2.110 de la Ciudad Autónoma de Buenos Aires aparece explicitada la "perspectiva de género" como uno de los principios del artículo 4. También la Ley ESI de la Provincia de Buenos Aires (№ 14.744) sancionada en el año 2015 establece la enseñanza de la sexualidad "desde la perspectiva de género, promoviendo el respeto a la diversidad y la no discriminación". Consideramos que este enfoque es el legado más importante que imprimió la participación de los movimientos de mujeres en su discusión pública previa y también en su elaboración. En la actualidad es uno de los 5 ejes que aparece con mayor fuerza en los dispositivos de formación inicial y capacitación docente.
} 
orientados principalmente a promover la autonomía y el cuidado de aspectos referidos a la salud sexual y reproductiva; a los distintos momentos de la reproducción humana desde todas las dimensiones (éticas, sociales, psicológicas y afectivas); a los métodos que previenen embarazos e infecciones, en especial el VIH. Asimismo, los contenidos enfatizan en el reconocimiento de los/as estudiantes como sujetos de derechos, con saberes, experiencias, y particularmente emociones propias que deben ser tenidos en cuenta.

Esto queda plasmado en la inclusión del contenido "El reconocimiento de emociones y sentimientos vinculados con la sexualidad humana y sus cambios, estableciendo su diferencia con la reproducción y genitalidad". Se puede ver de esta forma que la sexualidad no remite a un fin meramente reproductivo, a la vez que permite abordar la enseñanza de los genitales desde la dimensión del placer y sin la presunción de complementariedad basada en la reproducción heterosexual.

Por otro lado, aparece el siguiente contenido:

El conocimiento de las situaciones de riesgo o de violencia vinculadas con la sexualidad: distintas miradas sobre la problemática del aborto (como problema ético, de salud pública, moral, social, cultural y jurídico etc.), las enfermedades de transmisión sexual, el acoso sexual, el abuso y la violencia sexual, el maltrato, la explotación sexual y trata. (2008: 36)

Como es de presuponer, este contenido fue objeto de debate antes de ser finalmente incorporado. Si bien mantiene cierta neutralidad y en su formulación completa parece reducir el aborto a casos de violencia sexual, también significó un gran avance en cuanto a su visibilización como práctica recurrente y/o como problemática social. Resulta necesario destacar la inclusión de esta palabra, hasta no hace mucho innombrable en las escuelas, o tratada desde una mirada criminalizante (Bellucci, 2014).

El contenido "Conocimiento de los organismos protectores de derechos de su entorno (líneas telefónicas, programas específicos, centros de atención, etc.)" no sólo promueve la construcción colectiva de recursos disponibles para crear una red de ayuda, sino también visibiliza la responsabilidad del Estado en brindar herramientas que suelen ser muy demandadas desde la sociedad. En este mismo sentido, el ciclo orientador profundiza en la enseñanza de las dimensiones sociales de la sexualidad. El documento habilita así una mirada crítica sobre las prácticas de salud, y se expresa en contenidos cómo:

La indagación y análisis crítico sobre distintas concepciones sobre la salud y la sexualidad en el tiempo y en los distintos contextos [...] los mitos o creencias del sentido común en torno al cuerpo y la genitalidad [...] la construcción social e histórica del ideal de la belleza y del cuerpo para varones y mujeres.

Finalmente, el contenido "La comprensión de la sexualidad humana desde la perspectiva científica", también debe ser analizado a fondo a los fines de este trabajo. En una primera 
lectura, esta inclusión posiciona a la ESI en la defensa de la mirada científica como oposición a los discursos de ciertas instituciones religiosas ${ }^{6}$ que han elaborado materiales alternativos para contrarrestar el enfoque de la ley. La asignatura Biología es testigo de incontables debates entre las interpretaciones bíblicas referidas a los roles femeninos y masculinos, a la familia, así como también al origen de la vida y la evolución humana. $\mathrm{Si}$ consideramos la importante influencia del discurso religioso en la enseñanza de la sexualidad, podemos decir que esta inclusión de lo científico es también una conquista del movimiento de mujeres.

Sin embargo - y aquí está el punto central del argumento y la razón por la cual traemos los debates de la epistemología feminista- no podemos perder de vista que en los Lineamientos Curriculares, base de los contenidos mínimos obligatorios, no se explicita que la ciencia es un terreno de disputas de sentidos e intereses que se cuelan directamente en los contenidos escolares. La perspectiva científica, si bien es de utilidad para enfrentar el dogmatismo de la religión, está lejos de ubicarse en la neutralidad y en la garantía de "verdad sobre los cuerpos"

Un modo de problematizar esto es preguntarse ¿qué sucede con los "otros temas" clásicos del programa de Cs Naturales o Biología? La imagen clásica de la Evolución humana, tal como describe Harding, es sumamente anacrónica, eurocéntrica y misógina. El determinismo genético, como despliega Fox Keller(2000), se ha vuelto el núcleo duro más difícil de analizar para desmantelar el pensamiento determinístico. La enseñanza de los seres vivos, sin ir más lejos, es un terreno fértil para problematizar la clasificación y jerarquización arbitraria que desarrollaron Ixs científicos sobre la materialidad, incluyendo los cuerpos humanos. La extrema variedad de modos de reproducción y de mutaciones entre machos y hembras presente en las distintas especies representa una gran oportunidad para ahondar en un paradigma que no conceptualice al mundo natural como ordenado y fijo y binario, sino por el contrario, como diverso y en constante movimiento (González del Cerro y Busca, 2017)

En segundo lugar, ¿cómo se tradujeron los textos de la ESI a la práctica escolar? En el año 2015 se publicó un informe sobre "Balances y desafíos de la implementación de la ley ESI" (Faur et al 2015), elaborado por el Ministerio de Educación de la Nación. En él se analizan las instancias de capacitación docente llevadas a cabo desde el 2008 de modo cuantitativo y cualitativo, buscando dar cuenta de los alcances y los sentidos que aparecen sobre la

\footnotetext{
${ }^{6}$ Ejemplo reconocido de ello es el cuadernillo "Educar para el amor" que elaboró y distribuyó la Conferencia Episcopal Argentina en 2007 tras la sanción de la ESI y que pregonan una sexualidad basada en la abstinencia, la castidad y los roles naturales de varones y mujeres.

${ }^{7}$ Los desafíos de construir consensos desde un programa de alcance masivo en torno a las diversas lecturas sobre los cuerpos ameritan un análisis aparte, así como también requiere una rigurosa descripción de los distintos recursos elaborados y que en ocasiones dan cuenta de las transformaciones de miradas y énfasis al interior del propio PNESI desde su creación en 2009.
} 
política ESI. Aquí identificamos dos resultados que entendemos como relevantes para nuestro análisis.

En primer lugar, existe un acuerdo mayoritario entre docentes y directivos sobre el valor social de la educación sexual como responsabilidad de las instituciones educativas, sobre el enfoque integral que incluya distintas dimensiones de la sexualidad, y que su abordaje debe alcanzar todos los niveles educativos. Si retomamos los puntos de desacuerdo que manifestaron los sectores religiosos durante la sanción de la ley, vemos que en la actualidad se logró un consenso social sobre estos ejes (actores responsables, enfoques y destinatarios). Los puntos que siguen generando inquietudes en algunos casos son, según el informe, la no identificación de las discriminaciones a mujeres, la persistencia de cierta estigmatización de la diversidad sexual y la preocupación sobre el accionar frente a situaciones de abusos.

En segundo lugar, los resultados muestran que:

Ciencias Naturales es el área curricular en la que la absoluta mayoría de los directivos indica que se desarrollan contenidos de ESI con frecuencia (84\%). Seguidamente, si bien en proporciones más bajas, se ubica el área de Formación Ética y Ciudadana, y de Educación Física. (Faur et al 2015:102).

Así, en las escuelas de nuestro país Ixs docentes del área de biología o Cs. Naturales siguen siendo en las representaciones de la comunidad escolar Ixs responsables "naturales" de su implementación, lo que permite pensar que el modelo biomédico aún sigue siendo dominante cuando se lleva a la práctica la ESI.

Si volvemos a la propuesta de las pedagogas feministas antes citadas, nos preguntamos si no es estratégico pasar a otra fase, la de la politización de las asignaturas, sin redundar en una compensación cuantitativa. Es decir, no se trata de "agregar" nuevos contenidos de sexualidad al programa de las "otras" asignaturas, sino de revisar la construcción androcéntrica de los contenidos curriculares tradicionales, tal como es el caso de la enseñanza de la evolución humana en biología. Una hipótesis a seguir profundizando es que probablemente sea hora de enfocarse menos en antiguo silenciamiento que se supone la religión imponía para evitar la promiscuidad, o las resistencias por parte de la mirada moralizante de la sexualidad, y enfocarse más en la no cuestionada cristalización del pensamiento científico hegemónico (positivista y androcéntrico) como terreno más "cómodo" o estratégico para legitimar socialmente la ESI.

Tampoco se trata de inhabilitar el lenguaje científico, sino por el contrario, dar cuenta de lo que la acumulación de saberes de las distintas disciplinas científicas aporta para una mirada sobre la naturaleza que pueda dar cuenta del cambio y la diversidad de ésta, incluyendo en su enseñanza un análisis desde la epistemología crítica en general y feministas en particular. El campo de la didáctica de la biología local viene produciendo reflexiones en esta dirección, como lo hacen Massarini, Schnek, Piccinali, y Folguera, G. 
(2007) quienes elaboran una revisión histórica de la enseñanza de la biología en la Argentina y señalan la tardía introducción de la teoría evolutiva en la formación docente, obstaculizada en parte por presiones de sectores de la Iglesia católica. Apuntan a revalorizarla práctica de la investigación científica y tecnológica en vinculación con los objetivos del desarrollo social y a ensayar una alfabetización científica que vaya más allá de las interpretaciones reduccionistas de fenómenos aislados, dando lugar a la idea de complejidad e incertidumbre. En la misma línea los trabajos de González Galli, AdúrizBravo y Meinardi (2005) dan cuenta a partir de investigaciones en didáctica de la biología, del predominio de explicaciones teleológicas y finalistas que traen lxs estudiantes y la necesidad de abordar la enseñanza partiendo desde estas dificultades instaladas.

Asimismo Schnek (2011) da cuenta de que durante décadas los diseños curriculares priorizaron un enfoque predominantemente ecológico, que si bien fue importante, instaló una mirada reducida al vínculo entre estructura y función y dejó de lado los procesos de adaptación, abonando a lo que ella llama un imaginario "lamarkista" de la selección natural. La autora destaca asimismo la necesidad de des-romantizar la idea de evolucionismo lineal (al igual que lo hace Harding) así como también de armonía y perfección del mundo natural, y cita al propio Darwin:

Muchas veces sucede que los materiales de divulgación, por el hecho de entusiasmar al lector, apelan a las maravillas de la naturaleza. Sin embargo, como expresó Darwin, no debemos olvidar que no todas las disposiciones de la naturaleza son perfectas, ni mucho menos. La tendencia a pensar en la perfección se aleja de un concepto primordial en evolución: evolución no significa progreso, sino cambio. (Schnek 2011:28)

\section{Reflexiones finales}

Tanto la epistemología como la pedagogía feminista señalan los problemas de asumir como objetivos los saberes producidos y trasmitidos por científicxs y por docentes y estudiantes. Principalmente ponen en valor la capacidad narrativa, subjetiva, emotiva y singular de las personas que forman parte de un hecho educativo; hecho que involucra conocimientos elaborados en los distintos ámbitos culturales incluyendo los científicos. A partir de desplegar los tres proyectos feministas abordados a la luz de los debates del campo pedagógico, buscamos aportar a nuevos ejes de análisis para quienes vayan a observar las diversas modalidades posibles en las que se incorpora o traduce el feminismo en la educación, y también, reescribiendo nuevamente a Harding, sobre el papel de la educación en el feminismo.

Por otra parte, destacamos y festejamos los enormes avances que habilitaron la sanción y paulatina puesta en práctica de la ESI, ya que representa la "puerta de entrada" con la que contamos en la actualidad para introducir una posición crítica y explícitamente feminista del conocimiento en el ámbito escolar; ámbito que reconocemos como potente terreno de construcción de sentidos antipatriarcales. Sin embargo, consideramos que si no 
problematicemos como feministas el hecho de que seguimos "escudadas" bajo el paradigma científico, aun predominantemente androcéntrico, se corre el riesgo de terminar cristalizando esta autoridad epistémica como referencia última de nuestro accionar. Será necesario agudizar la capacidad de observar detenidamente el contexto de descubrimiento de los problemas a investigar (y enseñar) y las relaciones de fuerza religiosas, académicas, mediáticas y activistas de la coyuntura local actual para encontrar los modos de direccionar y profundizar una pedagogía de orientación feminista.

En el artículo nos preocupamos no tanto de la persistente legitimidad del área de ciencias en general- $y$ de las naturales en particular- con respecto a la educación sexual, sino por la pregunta sobre lo que esta asignatura escolar y la ciencia enseñada y producida en la órbita universitaria puede o debe reflexionar sobre sí misma a partir de sumar interrogantes históricos, políticos y epistemológicos, algo que la necesaria revisión curricular de la ESI promueve. El lugar de autoridad epistémica que suelen ocupar Ixs docentes de ciencias naturales en la institución, en tanto "especialistas" sobre lo natural, sobre los cuerpos y la sexualidad, puede entenderse como un riesgo (de cristalizar posiciones esencialistas bajo lo "científicamente comprobado" y patologizar lo "anormal"), pero también como una oportunidad para llevar adelante los objetivos, quizás lo más difíciles, que se propone la ESI con perspectiva de género. Gracias a la legitimidad antes mencionada, Ixs docentes de esta área corren con ventaja al momento de proponer talleres, charlas, consultorías para problematizar las diferencias a la luz de las propias experiencias, y también es una oportunidad para trabajar con contenidos éticos y habilitar la posibilidad de la incertidumbre. O por lo menos, retomando palabras de la propia Britzman (1999), tensionar algunas verdades instaladas, algo que generalmente Ixs docentes de biología no enfrentan. Maturana (1997) lo explicita cuando expresa que "he aceptado como problema legitimo para mi consideración como biólogo, temas y problemas [el lenguaje, las emociones y la ética] que para otros efectos podría decirse que no me pertenecen"(1997:41). Hemos intentado aquí rescatar y reconocer la interdisciplinaridad característica de los estudios de género y sus vertientes en dos campos que problematizan el conocimiento, para abrir nuevos interrogantes -y apropiaciones- en áreas disciplinares que por momentos parecerían considerarse ajenas a ellos.

\section{BIBLIOGRAFÍA}

Aisenstein, A. y Scharagrodsky, P. (2006): Tras las huellas de la educación física escolar argentina: cuerpo, género y pedagogía, 1880-1950. Buenos Aires, Argentina: Prometeo Libros Editorial.

Alonso G. y Zurbriggen, R. (2014): Transformando corporalidades: desbordes a la normalidad pedagógica. Educar em Revista, 53-69.

Apple, M. (1986): Ideología y currículo. Madrid, España: Akal. 
Baez, J. (2013): "La experiencia educativa "trans". Los modos de vivir el cuerpo sexuado de los/as/xs jóvenes en la escuela secundaria". Tesis doctoral en Ciencias de la Educación. Buenos Aires: Universidad de Buenos Aires, Facultad de Filosofía y Letras.

(2016): La inclusión de la educación sexual en las políticas públicas de América Latina. Los organismos internacionales y sus formas de intervención. En Revista Latinoamericana de Educación Comparada Vol 7, No 9, p. 71.

Bellucci, M. (2014): Historia de una desobediencia: aborto y feminismo. Buenos Aires, Argentina: Capital intelectual.

Bernstein, B. (1990): Poder, educación y conciencia. Barcelona, España: El Roure.

Bonder, G. (1994): “Mujer y Educación en América Latina: hacia la igualdad de oportunidades", en Revista Digital Iberoamericana de Educación, N 6- Género y Educación. [On Line] Disponible en: www.campus-oei.org/oeivirt/rie06.htm

Bourdieu, P. y Passeron, J.C. (1977): La reproducción .Barcelona, España: Laia.

Braidotti, R. (2004): Feminismo, diferencia sexual y subjetividad nómade. Barcelona, España: Editorial Gedisa.

Britzman, D. (1999): Curiosidade, sexualidade e curriculo en Lopes Louro, Guacira. O corpo educado. Pedagogias da sexualidade. Belo horizonte, Brasil: Autentica.

Chalmers, A. F (2000): ¿Qué es esa cosa llamada ciencia? Madrid, España: Siglo XXI

Da Cunha, M. (2015): “El currículum como Speculum" en Bach, A.M. (Coord.): Para una didáctica con perspectiva de género. Buenos Aires, Argentina: UNSAM.

Elizalde, S. (2006): “El androcentrismo en los estudios de juventud: efectos ideológicos y aperturas posibles" en Última década, Vol 14, No 25, p. 91-110.

Epstein, D. y Johnson, R. (2000): Sexualidades e institución escolar. Madrid, España: Ediciones Morata.

Faur, E., Gogna, M., y Binstock, G. (2015): La Educación Sexual Integral en la Argentina. Balances y desafíos de la implementación de la ley (2008-2015). Programa Nacional de Educación Sexual Integral.

Felitti, K (2011): “Educación sexual en la Argentina: políticas, creencias y diversidad en las aulas", en Antonio Marquet (coord.): Hegemonía y desestabilización: diez reflexiones en el campo de la cultura y la sexualidad. México DF: Fundación Arco Iris - Ediciones EON p. 113-133. 
Finocchio, S. (2009): "Las invenciones de la docencia en la Argentina (o de cómo la historia escolar transformó progresivamente a los enseñantes en sujetos resignados)" en Propuesta Educativa, No31, 41-53.

Fox Keller, E. (1991): Reflexiones sobre género y ciencia. Valencia España: Alfons el Magnànim.

(2000): Lenguaje y vida: metáforas de la biología en el siglo XX. Buenos Aires - Argentina: Ediciones Manantial.

Freire, P. (1970): Pedagogía del oprimido. Buenos Aires, Argentina: Tierra Nueva.

Giroux, H. (1992): Teoría y resistencia en educación. Buenos Aires, Argentina: Siglo XXI.

González del Cerro, C. y Busca, M. (2017): Más allá del sistema reproductor. Aportes para la enseñanza de la biología desde una perspectiva de género. Rosario, Argentina: Editorial Homo sapiens.

González Galli, L., Adúriz-Bravo, A., \& Meinardi, E. (2005): “El Modelo Cognitivo de Ciencia y los obstáculos en el aprendizaje de la Evolución Biológica”, en Enseñanza de las ciencias, (Extra), 1-6.

González García, M. y Pérez Sedeño, E. (2002): “Ciencia, Tecnología y Género". [On Line] Disponible en www.oei.es/revistactsi/numero2/varios2.htm

Haraway, D. J. (1995): Ciencia, cyborgs y mujeres. La reivindicación de la naturaleza. Madrid, España: Cátedra.

Harding S. (1996): Ciencia y feminismo. Madrid, España: Ediciones Morata.

Irigaray, L y Alonso, B. A. (1978): Speculum: espéculo de la otra mujer. Madrid, España: Saltés.

Kornblit, A., Sustas, S., Adasko D. (2013): “Concepciones sobre sexualidad y género en docentes de escuelas públicas argentinas", en Ciencia, docencia y tecnología, vol. 24, no 47, 47-78.

Korol, C. (2007): Hacia una pedagogía feminista: géneros y educación popular. Buenos Aires, Argentina: Editorial El Colectivo América Libre

(2016): Feminismos populares. Pedagogías y políticas. Buenos Aires, Argentina: Editorial El Colectivo América Libre

Lavigne, L. (2011): “Las sexualidades juveniles en la educación sexual integral”, en Elizalde, S. (coord.): Jóvenes en cuestión. Configuraciones de género y sexualidad en la cultura, p. 87-118. Buenos Aires, Argentina: Biblos. 
Lopes Louro, G. (1999): "Pedagogias da sexualidade", en LopesLouro ( comp): O corpo educado: pedagogias da sexualidade, p. 7-34. Belo Horizonte, Brasil : Autêntica.

Maffía, D. H. (2007): “Epistemología feminista: la subversión semiótica de las mujeres en la ciencia", en Revista venezolana de estudios de la mujer, Vol 12, No 28, p. 63-98.

Maher, F. y Thompson Tetreault, M.K. (1994): El aula feminista. Una mirada interna sobre cómo profesores y estudiantes están transformando la educación superior para una sociedad diversa. EEUU: EdBasicBooks.

Massarini, A., Schnek, A., Piccinali, R., y Folguera, G. (2007): "Democratizar el conocimiento científico: criterios y estrategias para un cambio en la enseñanza de las ciencias", en Cuarto Congreso Comunicación Social de la Ciencia. Cultura Científica y Cultura democrática. 8 p.

Maturana, H. (1997): Emociones y lenguaje en educación y política. Santiago, Chile: Dolmen.

McIntosh, P. (1990): "Interactive phases of curricular and personal re-vision with regard to race", en Adams and Welsch (Editors): Multicultural Prism: Voices from the Field. Volume 3. Wellesley College, Center for Research on Women.

Molina, G. (2014): Género y sexualidades entre estudiantes secundarios. Un estudio etnográfico en escuelas cordobesas. Buenos Aires: IDES-Miño y Dávila Editores.

Morgade, G.,y Alonso, G. (2008): Cuerpos y sexualidades en la escuela. Buenos Aires: Paidós

Morgade, G. (1995) “Notas sobre la contradictoria relación entre la nueva sociología de la educación y el género", en Revista Mora, № 1. Revista del Área Interdisciplinaria de Estudios de la Mujer, p. 67-70. Fac. de Filosofía y Letras -UBA, Buenos Aires.

(2006): "Educación en la sexualidad desde el enfoque de género", en AAVV Novedades Educativas №184, p. 40 - 44. NOVEDUC, Buenos Aires.

- (2010): Mujeres que dirigen poniendo el cuerpo: poder, autoridad y género en la escuela primaria. Buenos Aires, Argentina: Noveduc.

-- (coord.) (2011): Toda educación es sexual. Buenos Aires, Argentina: La Crujía

(2013): “Notas epistemológicas desde una investigación feminista sobre educación sexual", en Revista da FAEEBA-Educação e Contemporaneidade, 22(40).

Ochoa, Luz Maceira (2008): El sueño y la práctica de sí. Pedagogía feminista: una propuesta. México D.F., México: El Colegio de México, Centro de Estudios Sociológicos, Programa Interdisciplinario de Estudios de la Mujer. 
Pacheco Ladrón de Guevara, L. (2010): El sexo de la ciencia. México DF, México: Universidad Autónoma de Nayarit, México.

Péchin, Juan (2012): “Géneros, sexualidades y resistencias políticas a la normalización. Etnografía sobre procesos identitarios en/desde la escolaridad del siglo XXI en Buenos Aires". Tesis de doctorado. Buenos Aires: Universidad de Buenos Aires, Facultad de Filosofía y Letras.

Pérez Sedeño, E. (1998): “Factores contextuales, tecnología y valores: ¿desde la periferia?", en Contrastes.

Pineau, P., Dussel, I.yCaruso, M. (2001): La escuela como máquina de educar. Buenos Aires, Argentina: Ed. Paidos.

Puiggrós, A. (2014): “Educación y sociedad en América Latina de fin de siglo: del liberalismo al neoliberalismo pedagógico", en Estudios Interdisciplinarios de América Latina y el Caribe, 10(1).

Rigal, L. (2011): “Lo implícito y lo explícito en los componentes pedagógicos de las teorías críticas en educación", en Hilert, f., Ameijeiras, Mj \& Graziano, N.[comp.]: La mirada pedagógica para el siglo XXI: teoría, temas y prácticas en cuestión. Reflexiones de un encuentro. Buenos Aires, Argentina: Editorial de la Facultad de Filosofía y Letras-Universidad de Bs. As. Secretaría de Investigación y Posgrado.

Scharagrodsky, P. (2008): "El cuerpo en la escuela", en Revista Explora. Ministerio de Educación Ciencia y Tecnología, Presidencia de la Nación.

Schiebinger, L. L. (2004): ¿Tiene sexo la mente?: las mujeres en los orígenes de la ciencia moderna. Valencia, España: Universitat de València.

Shor, I. (1980): Critical teaching and everyday life. Boston, USA: South End Press,

Terigi, F. (2000): Curriculum. Buenos Aires, Argentina: Santillana.

Tomasini, Marina (2011): “Jóvenes en la escuela: hacer género en las peleas". Actas IV Coloquio Interdisciplinario Internacional Educación, sexualidades y relaciones de género. Facultad de Filosofía y Letras. Buenos Aires.

Willis P. (1983): Aprendiendo a trabajar. Madrid, España: Akal

\section{Documentos curriculares citados}

Ministerio de Educación de la Nación (2008): Lineamientos Curriculares para la Educación Sexual Integral. 
Revista Punto Género № 8. Diciembre de 2017

ISSN 0719-0417 / 55 - 77

Ministerio de Educación de la Nación (2010): Cuadernos ESI para el aula. Nivel Secundario I.

Ministerio de Educación de la Nación (2012): "Cuadernos ESI para el aula. Nivel Secundario II.

Ministerio de Educación de la Ciudad Autónoma de Buenos Aires (2007): Lineamientos Curriculares para la Educación Sexual Integral. Nivel Secundario. 